\title{
Postexercise hypotension and autonomic modulation response after full versus split body resistance exercise in trained men
}

\author{
Marcelo Conrado de Freitas', Ana Laura Ricci-Vitor², Giovanni Henrique Quizzini', João Vitor N. S. de Oliveira3, \\ Luiz Carlos M. Vanderlei², Fabio Santos Lira ${ }^{3}$, Fabrício Eduardo Rossi ${ }^{4, *}$ \\ 'Skeletal Muscle Assessment Laboratory, School of Technology and Sciences, Department of Physical Education, São Paulo State University, Presidente Prudente, Brazil \\ ²Department of Physical Therapy, São Paulo State University (UNESP), Presidente Prudente, Brazil \\ ${ }^{3}$ Exercise and Immunometabolism Research Group, Department of Physical Education, São Paulo State University (UNESP), Presidente Prudente, Brazil \\ ${ }^{4}$ Immunometabolism of Skeletal Muscle and Exercise Research Group, Department of Physical Education, Federal University of Piauí (UFPI), Teresina, Brazil
}

To investigate the effects of full versus split body resistance exercise on postexercise hypotension and autonomic modulation in trained men. Sixteen resistance recreationally trained males (age, $24.9 \pm 5.3$ years) performed three randomized trials: upper body (UB), lower body (LB), and full body (FB) conditions. Blood pressure and heart rate variability were collected at rest, immediately postexercise, post-10, and post-30 min during recovery. For systolic blood pressure, delta (30 min minus rest) was lower for the FB condition compared to the UB $(-10.1 \pm 7.4$ $\mathrm{mmHg}[\mathrm{FB}]$ vs. $-3.3 \pm 12.6 \mathrm{mmHg}$ [LB] vs. $-1.9 \pm 8.1 \mathrm{mmHg}[\mathrm{UB}], P=0.004)$. For diastolic blood pressure $(-8.2 \pm 10.9 \mathrm{mmHg}$ [FB] vs. $-1.5 \pm 9.8 \mathrm{mmHg}$ [LB] vs. $-8.7 \pm 11.4 \mathrm{mmHg}[\mathrm{UB}], P=0.038)$ and mean blood pressure delta during recovery $(-11.7 \pm 14 \mathrm{mmHg}[\mathrm{FB}]$ vs. $-2.2 \pm 10.6 \mathrm{mmHg}[\mathrm{LB}]$ vs. $-5.2 \pm 6.8 \mathrm{mmHg}[\mathrm{UB}], P=0.045)$, there were statistically significant lower values in the FB condition in relation to the LB condition. Regarding heart rate variability, there were no significant differences between conditions, however, the square root of the mean squared difference between adjacent $R R$ intervals presented an increase until post-30 during recovery $(P<0.001)$, and there was a higher values of low frequency and lower values of high frequency across time $(P<0.001)$. Postexercise hypotension was influenced by the amount of muscle mass involved in a bout of resistance exercise, with the FB condition inducing lower systolic blood pressure in relation to the UB after exercise, as well as a great decrease in postexercise diastolic and mean blood pressure compared with the LB. The autonomic modulation response was similar between conditions during recovery.

Keywords: Strength exercise, Blood pressure, Autonomic nervous system

\section{INTRODUCTION}

Resistance exercise (RE) is often used as a nonpharmacological tool for treatment and prevention of cardiovascular diseases (Pescatello et al., 2004), leading to improvements in musculoskeletal, metabolic, and cardiovascular systems (Hurley and Roth, 2000). It has been reported that acute RE can reduce blood pressure (Pescatello et al., 2004), a condition denominated post-exercise hypotension (PEH), and has an essential contribution in controlling high blood pressure (Gomes Anunciação and Doederlein Polito,
2011). Previous studies have investigated the effects of an acute RE program on blood pressure response, showing that intensity, volume, rest interval between sets, and amount of active muscle mass in RE can influence the magnitude or duration of PEH (de Salles et al., 2010; Figueiredo et al., 2015; Polito and Farinatti, 2009; Simão et al., 2005). Besides this, blood pressure responses are modulated by the autonomic nervous system (Joyner and Casey, 2015; Kulics et al., 1999).

The autonomic nervous system can be evaluated using heart rate variability, an accepted method to analyze changes which in-
${ }^{*}$ Corresponding author: Fabrício Eduardo Rossi

(iD) https://orcid.org/0000-0002-0594-2529

Department of Physical Education, Federal University of Piauí (UFPI), "Ministro Petrônio Portella" Campus, Teresina-PI 64049-550, Brazil

Tel: +86-3215-5525, Fax: +86-3215-55264391, E-mail: fabriciorossi@ufpi.edu.br Received: March 5, 2018 / Accepted: April 26, 2018
This is an Open Access article distributed under the terms of the Creative Commons Attribution Non-Commercial License (http://creativecommons.org/licenses/by-nc/4.0/) which permits unrestricted non-commercial use, distribution, and reproduction in any medium, provided the original work is properly cited. 
fluence the cardiovascular system (Borresen and Lambert, 2008). Previous studies have demonstrated that heart rate variability can be used to evaluate cardiovascular overload and stress induced by exercise (Al Haddad et al., 2011; Stanley et al., 2013). Heart rate variability indices can also be applied to measure autonomic modulation during recovery as well as parasympathetic reactivation after a bout of exercise (Kiviniemi et al., 2010; Stanley et al., 2013). It has been reported that RE can drastically reduce parasympathetic modulation (Heffernan et al., 2006; Heffernan et al., 2008) and reductions in heart rate variability are related with cardiovascular events such as ventricular arrhythmias and sudden cardiac death as well as increased mortality risk (Pokorný et al., 2011; Vinik et al., 2011; Volders, 2010). In this sense, evaluation of autonomic modulation after acute bouts of RE with different program structures may have great clinical implications on RE prescription for healthy and cardiac patients.

Regarding investigation of the effects of RE with different limbs on PEH. Polito and Farinatti (2009) demonstrated that significant reductions in systolic blood pressure (SBP) and mean blood pressure (MBP) only occur in lower body RE (leg extension, 10 sets with 10 repetitions), without significant results in upper body RE (biceps curl, 10 sets with 10 repetitions), suggesting the amount of active muscle mass during RE has an influence on $\mathrm{PEH}$. To our knowledge, only one study compared the influence of acute full versus upper and lower body routines on heart rate variability in trained men and woman. Kingsley et al. (2014) observed that postexercise autonomic modulation was similar between conditions, while $25 \mathrm{~min}$ after exercise parasympathetic modulation was lower compared with rest. However, the study conducted by Kingsley et al. (2014) did not investigate blood pressure response, as the comparison between full versus split body routines on PEH is less explored in the literature.

From a practical point of view, it is necessary to understand the influence of RE with different routines (full or split body) on PEH and heart rate variability, since the results of this study could be used to direct the prescription of RE for blood pressure control. Thus, the purposes of this study were to investigate the effects of full versus split body RE on PEH and autonomic modulation in resistance-trained men.

\section{MATERIALS AND METHODS}

\section{Participants}

Sixteen recreationally resistance trained males (age, $24.9 \pm 5.3$ years; body weight, $78.5 \pm 11.9 \mathrm{~kg}$; height, $179.0 \pm 8.7 \mathrm{~cm}$ ) with a minimal 6 months of resistance training experience (experience, $4.5 \pm 2.8$ years; weekly frequency training, $4.1 \pm 0.8$ days) were recruited for this study. The inclusion criteria were: (a) aged between 20 and 30 years; (b) participates in regimented strength training $\geq 6$ months (American College of Sports Medicine, 2006); (c) had not used any ergogenic substance or medicine for at least 6 mo Lichtenstein et al. (2006) nths prior to the study; (d) no smoking or alcohol use; (e) no contraindications involving the cardiovascular system. The study was approved by the Ethics in Research Group of the University of São Judas, São Paulo-SP, Brazil (protocol number: 66523717. 2.0000.0089) and the research was conducted according to the 2008 Revision of the Declaration of Helsinki. All participants signed a consent form and were informed about the purpose of the study and the possible risks.

\section{Experimental design}

This study used a randomized and cross-over design. Subjects completed six experimental trials. The first visit was used to determine anthropometric measurements to characterize the sample. In the following two visits, one maximum repetition (1RM) test was performed for upper or lower body separated by $72 \mathrm{hr}$. In the fourth, fifth, and sixth visits, the participants randomly completed three experimental trials (full, upper, or lower body RE) separated by one week. All trials were performed at the same time (6:30 a.m. to 9:30 a.m.) to ensure chronobiological control. Heart rate variability and blood pressure were analyzed at rest in the supine position, immediately after the acute bout of RE, according to the experimental design shown in Fig. 1.

\section{Anthropometric measurements and dietary intake assessment}

Electronic scale (Filizola PL 50, Filizzola Ltda., São Paulo, Brazil) with a precision of $0.1 \mathrm{~kg}$ was used to determine body weight and a fixed stadiometer of the Sanny brand, (Sanny brand, American Medical of Brazil Ltda, São Paulo, Brazil) with an accuracy of $0.1 \mathrm{~cm}$ and length of $2.20 \mathrm{~m}$ was used to measured height of the

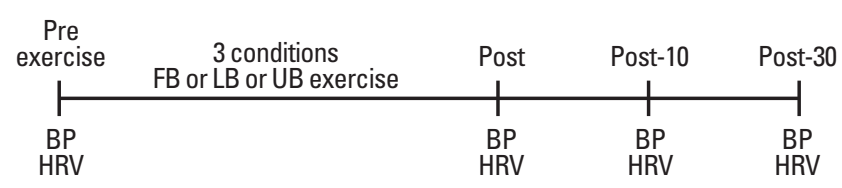

Fig. 1. Experimental design. BP, blood pressure; HRV, heart rate variability; $F B$, full body condition, 18 sets at $65 \%$ of $1 \mathrm{RM}, 90$ sec of rest; $L B$, lower body condition, 18 sets at $65 \%$ of $1 \mathrm{RM}, 90$ sec of rest; UB, upper body condition, 18 sets at $65 \%$ of $1 \mathrm{RM}, 90 \mathrm{sec}$ of rest. 
participants. The participants were positioning with their backs, shoulder blades, buttocks, and heels touching the stadiometer and they were wearing light clothing during assessment.

To guarantee participants presented equivalent conditions regarding energy, food questionnaires were distributed to all participants to record food intake for 3 days prior to each trial and a breakfast was provided to participants every day before the workout (1 hr $30 \mathrm{~min}$ ), consisting of 15\%-20\% protein, $50 \%-60 \%$ carbohydrates, and 25\%-30\% fat, according to the American Heart Association (Lichtenstein et al., 2006). Participants were instructed by a nutritionist as to how to complete the dietary questionnaires. All questionnaires were analyzed for total kilocalorie and macronutrient intake, averaged for the three days of trials to ensure that dietary intake was similar between experimental trials. The software (Software - Dietpro version 5.8, Nutrition Software, Viçosa, Brazil) utilized the database of Brazilian food composition table (TACO) to calculate dietary intake.

\section{Strength test and RE protocol}

Initially, the participants completed two sessions to become acquainted with the equipment and perform strength test procedures. A warm-up was performed prior to 1RM testing, which consisted of 5 min of walking and 1 set of 10 repetitions at approximately $50 \%$ of the $1 \mathrm{RM}$. The load was increased gradually $(10 \%-15 \%)$ during the test until the participants were no longer able to perform the entire movement, and 3-5 attempts were allowed as previously described (Rossi et al., 2016). In a randomized manner, the subjects performed two 1RM tests separated by $72 \mathrm{hr}$ : lower body ( $45^{\circ}$ leg press, leg extension, and leg curl) or upper body (bench press, T-bar row and elbow curl), respectively.

The subjects performed three randomized exercise sessions one week after the 1RM test. For the lower body, participants performed 6 sets at $65 \%$ of $1 \mathrm{RM}$ in the $45^{\circ}$ leg press, leg extension, and leg curl, respectively, for the upper body condition, they performed 6 sets at $65 \%$ of $1 \mathrm{RM}$ in the bench press, T-bar row, and elbow curl, respectively, and for the full body condition, the subjects performed 3 sets at $65 \%$ of $1 \mathrm{RM}$ in both lower and upper body routines, in this sequence. For all sequences, $90 \mathrm{sec}$ of rest was allowed between trials and exercises and all routines were directly supervised by the research team to ensure proper performance of the respective routines.

\section{Autonomic modulation and blood pressure measurements}

Autonomic modulation was recorded at rest and during recovery (30 $\mathrm{min})$, through heart rate variability expressed by calculat- ed indices in the time and frequency domains. Subjects were instructed to remain in silence, awake, at rest, breathing spontaneously in the supine position before and after the exercise routines, according with Kingsley et al. (2014). After the procedures had been explained, an elastic strap was placed on the subject at the height of the xiphoid process and a heart rate receptor on the wrist (Polar Electro, model RS800, from Polar Electro Oy, Kempele, Finland). This equipment has been previously validated to record beat-to-beat heart rate and heart rate variability analysis (Barbosa et al., 2016).

The temporal recording of the interval between consecutive cardiac beats (interval RR) was submitted to digital filtering using Polar software complemented by manual filtering. Only sets with more than $95 \%$ sinoatrial node beats were included in this study (Vanderlei et al., 2009). Next, intervals of five minutes with a minimum of $256 \mathrm{RR}$ intervals were analyzed and selected in Kubios software (Biosignal Analysis and Medical Image Group, Department of Physics, University of Kuopio, Kuopio, Finland) (Vanderlei et al., 2009).

For the time domain, the analysis included the square root of the mean squared difference between adjacent $R R$ intervals (RMSSD) and standard deviation of all normal RR intervals (SDNN). For the frequency domain, the analysis included spectral components of low frequency (LF: frequency between 0.04 and $0.15 \mathrm{~Hz}$ ) and high frequency (HF: frequency between 0.15 to 0.4 $\mathrm{Hz}$ ) expressed in normalized units (nu). Spectral analysis was calculated using Fast Fourier Transform (Vanderlei et al., 2008).

Physiologically, RMSSD and HF represents the parasympathetic component of the autonomic nervous system in the time and frequency domain respectively (Vanderlei et al., 2008). The SDNN, as a temporal index, represents global variability while LF index, from the frequency domain, represents the action of both components parasympathetic but with predominance of sympathetic branch (Vanderlei et al., 2008).

Blood pressure was recorded at rest, immediately postexercise, post-10, and post-30 after exercise. The systolic and diastolic blood pressure (DBP) were measured using an automatic blood pressure monitor (Omron Healthcare brand, Inc., Intellisense, Model HEM 742 INT, Bannockburn, IL, USA), previously validated (Coleman et al., 2005). The MBP was calculated using $(\mathrm{MBP}=\mathrm{SBP}+[2 \times \mathrm{DBP}] / 3)$.

\section{Statistical analyses}

A $3 \times 4$ (condition $\times$ time) repeated measures analysis of variance (RMANOVA) with the Bonferroni adjustment for multiple com- 
parisons was used to compare blood pressure and heart rate variability in the three experimental conditions across time. A RMANOVA was used to analyze the absolute differences (delta $=$ post -30 min value minus rest) for SBP, DBP, and MBP, and indices of heart rate variability. Mauchly's sphericity test was used to test this assumption, and a Greenhouse-Geisser correction applied when necessary. The effect sizes were calculated as the mean rest to postexercise change divided by the pooled rest standard deviation, whereby a value of $>0.20$ was considered small, $>0.50$ moderate, and $>0.80$ large and effect sizes for ANOVA were calculated using partial eta squared $\left(\eta^{2}\right)$ for time. Statistical significance was set at $P<0.05$. All analyses were performed using the SPSS ver. 10 (SPSS Inc., Chicago, IL, USA) and data are reported as means and standard deviation.

\section{RESULTS}

Table 1 shows the mean and standard deviation values for 24-hr dietary intake and macronutrient intake averaged across three days for each experimental trial as well as performance comparisons. There were no statistically significant differences between

Table 1. Comparison of dietary intake in the three experimental trials

\begin{tabular}{lcccc}
\hline Dietary Intake & Trial-1 & Trial-2 & Trial-3 & $P$-value \\
\hline Carbohydrate $(\mathrm{g})$ & $288.5 \pm 127.4$ & $275.6 \pm 115.5$ & $259 \pm 115.7$ & 0.192 \\
Protein $(\mathrm{g})$ & $124.5 \pm 54.3$ & $123 \pm 29.4$ & $111.1 \pm 31.7$ & 0.431 \\
Lipid $(\mathrm{g})$ & $69.6 \pm 22.5$ & $71.3 \pm 25.4$ & $78.2 \pm 32.2$ & 0.479 \\
Total intake (kcal) & $2,283 \pm 578.3$ & $2,236 \pm 558.6$ & $2,185 \pm 581.2$ & 0.716
\end{tabular}

Values are presented as mean \pm standard deviation. any trials for dietary intake.

Table 2 shows the comparisons of blood pressure in the three experimental conditions and Fig. 2 presents the differences in SBP, DBP, and MBP delta during recovery.

For SBP, there was a main effect of time $(F=8.055, P<0.001$, $\left.\eta^{2}=0.42\right)$, with a statistically significant interaction $(F=2.213$, $P<0.014)$. The post hoc analysis showed hypotension at post-10 and post-30 min in relation to immediately Postexercise but no differences between conditions were verified $(F=2.321, P=0.121)$.

Table 2. Comparison of blood pressure in the three experimental conditions

\begin{tabular}{|c|c|c|c|c|c|}
\hline Variable & Rest & Post & Post-10 & Post-30 & $\begin{array}{c}\text { Effect } \\
\text { size }\end{array}$ \\
\hline \multicolumn{6}{|l|}{ SBP (mmHg) } \\
\hline Upper body & $127.3 \pm 8.6$ & $137.6 \pm 13.1$ & $125.7 \pm 10.4^{b l}$ & $125.4 \pm 6.9^{b l}$ & 0.25 \\
\hline Full body & $129.3 \pm 11.8$ & $130.6 \pm 23.7$ & $119.2 \pm 20.1^{b)}$ & $119.3 \pm 9.5^{b)}$ & 0.94 \\
\hline Lower body & $128.1 \pm 9.4$ & $137.7 \pm 15.6$ & $122.3 \pm 8.5^{b /}$ & $124.8 \pm 11.3^{b)}$ & 0.32 \\
\hline \multicolumn{6}{|c|}{$\mathrm{DBP}(\mathrm{mmHg})$} \\
\hline Upper body & $69.2 \pm 9.7$ & $65.2 \pm 10.4$ & $57.8 \pm 10.7^{2 a, *}$ & $60.6 \pm 12.2^{\text {a) }}$ & 0.70 \\
\hline Full body & $66.8 \pm 10.2$ & $66.8 \pm 10.2$ & $58.0 \pm 10.9^{a, b, b} *$ & $57.8 \pm 7.6^{\mathrm{a}, \mathrm{b}, \mathrm{l}, \pm}$ & 1.01 \\
\hline Lower body & $68.4 \pm 11.2$ & $69.0 \pm 5.7$ & $66.2 \pm 7.3$ & $66.2 \pm 7.9$ & 0.23 \\
\hline \multicolumn{6}{|l|}{$\mathrm{MBP}(\mathrm{mmHg})$} \\
\hline Upper body & $88.1 \pm 7.0$ & $88.9 \pm 7.2$ & $80.4 \pm 6.3^{\mathrm{a}, \mathrm{b})}$ & $82.2 \pm 8.9^{b)}$ & 0.74 \\
\hline Full body & $87.6 \pm 7.8$ & $88.1 \pm 11.6$ & $78.4 \pm 9.8^{\mathrm{a}, \mathrm{bl}}$ & $75.8 \pm 12.2^{b)}$ & 1.18 \\
\hline Lower body & $88.3 \pm 8.6$ & $91.9 \pm 5.4$ & $\left.84.9 \pm 6.8^{\mathrm{a}, \mathrm{b}}\right)$ & $85.7 \pm 7.1^{b)}$ & 0.33 \\
\hline
\end{tabular}

Values are presented as mean \pm standard deviation.

SBP, systolic blood pressure; DBP, diastolic blood pressure; MBP, mean blood pressure.

${ }^{\text {al }}$ Significant difference from rest. ${ }^{b}$ Significant difference from postexercise.

*Significant difference from lower body. "Significant difference between full and lower body.

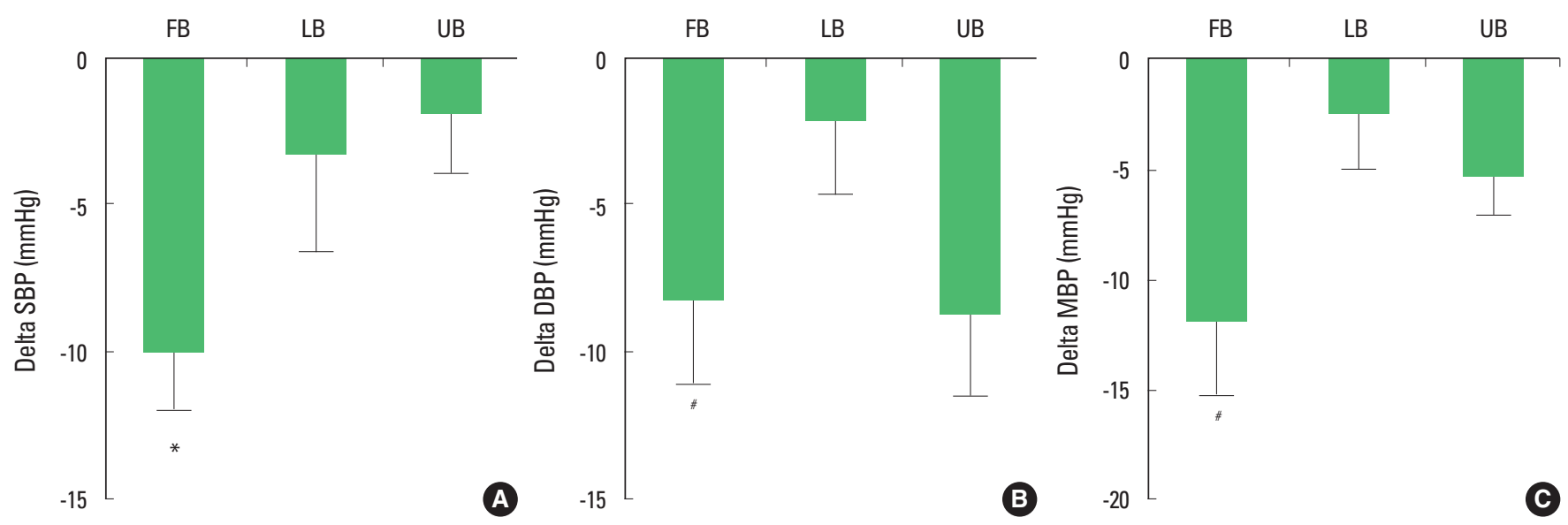

Fig. 2. Comparison of systolic, diastolic, and mean blood pressure delta (post-30 minus rest) during recovery. Values are expressed as mean \pm standard deviation. (A) Systolic blood pressure. (B) Diastolic blood pressure. (C) Mean blood pressure. *Statistically significant differences between full and upper body conditions. " $S$ tatistically significant differences between full and lower body conditions. 

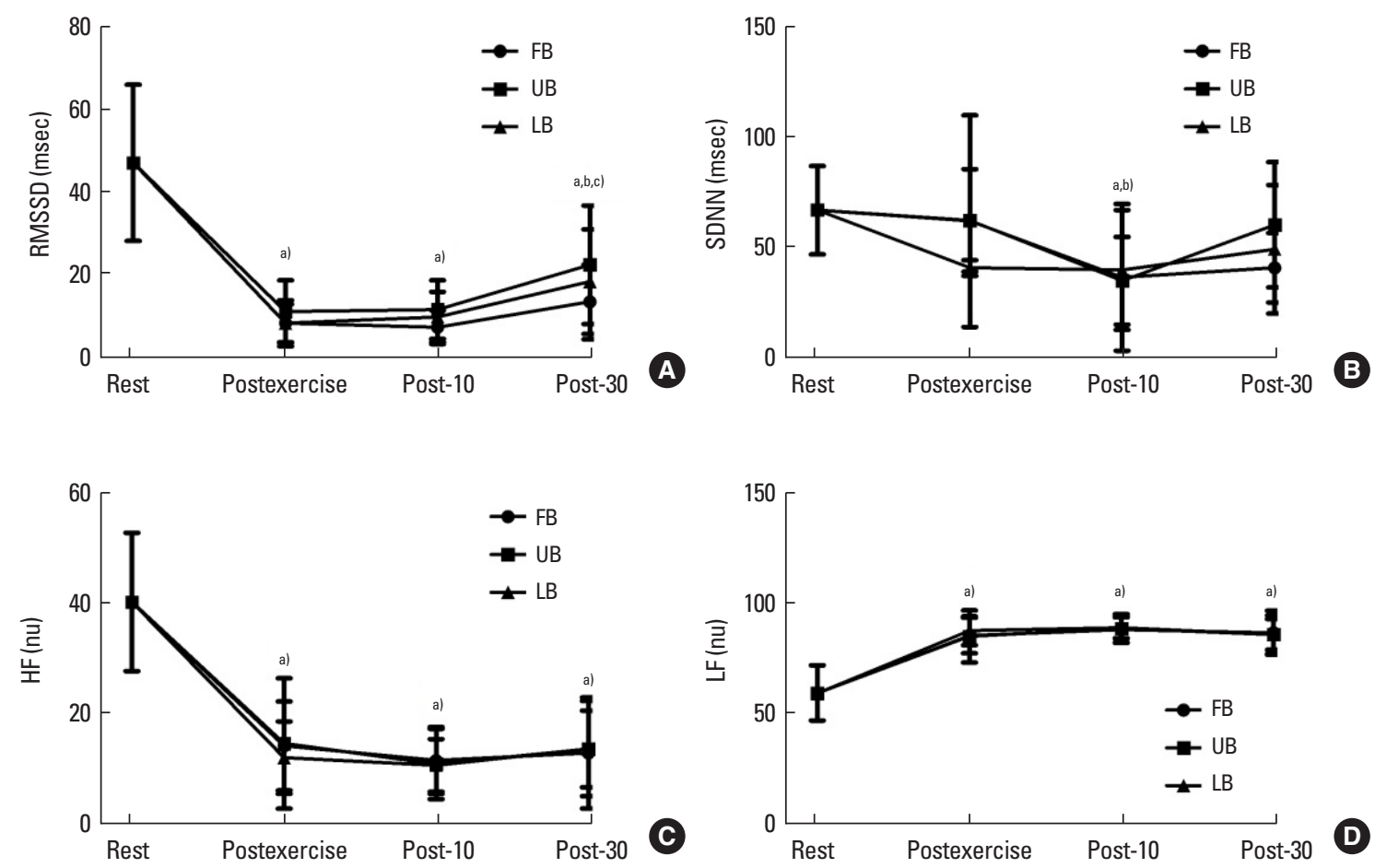

Fig. 3. Comparison of heart rate variability in the three experimental conditions. (A) The root mean square of successive differences between normal intervals (RMSSD) of consecutive cardiac beats, (B) the standard deviation of normal-to-normal intervals (SDNN), (C) high frequency (HF), (D) low frequency (LF), nu, normalized units. ${ }^{\text {al All }}$ condition were significantly different from rest. ${ }^{b /} \mathrm{All}$ condition were significantly different from postexercise. ${ }^{\mathrm{c}} \mathrm{All}$ condition were significantly different from post-10.

However, when analyzing the SBP delta, there was a significantly lower SBP for the FB condition compared to the UB condition $(P=0.004)$. Effect sizes were large for $\mathrm{FB}(0.94)$ and small for $\mathrm{LB}$ (0.32) and $\mathrm{UB}(0.25)$.

Regarding DBP, there was a main effect of time $(F=3.414, P=$ $\left.0.006, \eta^{2}=0.25\right)$ and a statistically significant difference between conditions $(F=6.121, P=0.008)$, but no significant interaction $(P>0.05)$. Delta analysis showed lower DBP in the FB condition in relation to the $\mathrm{LB}$ condition $(P=0.038)$. Effect sizes were large for FB (1.01), ranged from moderate to large for UB (0.70), and were small for $\mathrm{LB}(0.23)$.

For MBP, there was a main effect of time $(F=6.965, P<0.001$, $\left.\eta^{2}=0.38\right)$ and a statistically significant difference between conditions $(F=7.966, P=0.002)$, however, no significant interaction was observed $(P>0.05)$. In addition, the MBP delta verified lower values for the $\mathrm{FB}$ condition compared to the $\mathrm{LB}$ condition $(P=0.045)$. Effect sizes were large for FB (1.18), ranged from moderate to large for UB (0.74), and were small for LB $(0.33)$.

Fig. 3 shows the comparisons of heart rate variability in the three experimental conditions. For SDNN (msec), there was a main effect of time $\left(F=5.796, P=0.007, \eta^{2}=0.345\right)$ with significantly lower values at post-10 and immediately postexercise in re- lation to rest $(P<0.05)$.

For RMSSD, there was a main effect of time $(F=42.297, P<$ $\left.0.001, \eta^{2}=0.794\right)$ with lower values across time compared to rest and increased values post-30 min of recovery in relation to immediately postexercise and post-10, although maintained at lower values than at rest $(P<0.001)$.

For LF (nu), there was a main effect of time $(F=50.969, P<$ $\left.0.001, \eta^{2}=0.822\right)$ with higher values for all conditions compared to rest $(P<0.001)$. For HF (nu) there was a main effect of time $\left(F=51.074, P<0.001, \eta^{2}=0.823\right)$ with lower HF immediately postexercise, post-10, and post-30 in relation to rest $(P<0.001)$. For all indices analyzed, there were no significant differences between conditions and no significant interaction. Furthermore, when heart rate variability delta was verified during recovery, there were no significant changes in the $\operatorname{SDNN}(F=3.022, P=$ $\left.0.069, \eta^{2}=0.216\right), \operatorname{RMSSD}\left(F=2.007, P=0.158, \eta^{2}=0.154\right)$, or $\mathrm{LF}$ and $\mathrm{HF}\left(F=1.316, P=0.288, \eta^{2}=0.10\right)$.

\section{DISCUSSION}

The main findings of this study were that a bout of FB RE induced greater PEH than split body RE, since SBP post-30 min of 
exercise was lower in the FB compared with UB condition and there was a greater reduction in diastolic and MBP after exercise for FB in relation to LB. Regarding heart rate variability, participants presented physiological responses with no differences between the conditions, and a progressive increase in parasympathetic modulation during recovery was observed, in addition to which after $30 \mathrm{~min}$ of exercise sympathetic predominance persisted compared to rest.

Investigation into the influence of full versus split body resistance training on PEH is little explored in the literature. Polito and Farinatti (2009) analyzed the effects of upper versus lower body RE (biceps curl vs. leg extension) with 10 repetitions on $\mathrm{PEH}$. The results showed that only lower body exercise induced significant reductions in the systolic and MBP but no differences were observed between upper and lower body exercise for DBP, however, the authors did not compare the effects with the full body condition. On the other hand, in the present study, we showed that the FB condition induced greater $\mathrm{PEH}$ than both split body conditions; thus, our results suggested that the amount of muscle mass involved in a bout of RE can induce different $\mathrm{PEH}$ responses.

Clinically, these results have an important practical application, as they could be used to direct the prescription of RE for PEH, using FB training. It has been reported that maintenance of vasodilatation after exercise may influence, in part, PEH (Gomes Anunciação and Doederlein Polito, 2011; Halliwill, 2001). The possible mechanism by which RE with larger muscle mass induced higher PEH could be attributed to the great amount of muscle mass in a condition of vasodilation, leading to a reduction in vascular resistance (Halliwill, 2001; Mortensen and Saltin, 2014). In support, the increased blood flow to muscle during exercise can be potentiated by a higher amount of active muscle mass (Joyner and Casey, 2015). We hypothesize that the FB routine created an increase in the need for blood flow in the large amount of active muscles, which led to higher vasodilatation in both upper and lower regions, favoring the magnitude of $\mathrm{PEH}$.

Furthermore, the results showed that in the sessions involving UB exercise there was a significant reduction in DBP. Supporting our results, Drouet et al. (2017) showed that upper (bench press, lat pull down, and seated military press) and lower body (back squat, knee extension, and knee curl) RE lead to a reduction in SBP after 50 and $60 \mathrm{~min}$ in prehypertensive men, but a significant reduction in DBP following exercise was observed only in upper body exercise. Di Blasio et al. (2009) found that arm exercise (arm crank ergometer) provided a higher reduction in DBP in sedentary males after exercise compared with leg exercise (elliptical trainer) with 35-min duration at 55\%-60\% heart rate reserve. In addition, in our study, the FB and UB routines induced a greater decrease in DBP than the LB condition. However, further studies are necessary to investigate the mechanism by which the $\mathrm{RE}$ involving upper body region can decrease DBP.

Investigations into the influence of $\mathrm{RE}$ with different structures on heart rate variability response are necessary to establish an exercise program for patients at elevated cardiovascular risk. Kingsley et al. (2014) investigated the effects of full (leg extension, leg curl, seated row, and chest press) versus upper (seated row and chest press) and lower (leg extension and leg curl) body routines with three sets of 10 repetitions on heart rate variability indices in resistance-trained individuals. The authors observed that postexercise autonomic modulation was similar between conditions, generating a reduction in parasympathetic indices after $25 \mathrm{~min}$ of exercise. In accordance, our results showed a progressive decrease in parasympathetic modulation, and a sympathetic predominance that persisted until $30 \mathrm{~min}$ during recovery, with no significant differences in the evaluated indices was observed between conditions. Given that heart rate variability also can be used to evaluate cardiovascular overload and stress induced by exercise (Borresen and Lambert, 2008; Stanley et al., 2013) and reductions in heart rate variability are related with cardiovascular events such as ventricular arrhythmias and sudden cardiac death as well as increased mortality risk (Pokorný et al., 2011; Vinik et al., 2011; Volders, 2010), our results demonstrated that FB routine may be used to decrease blood pressure after exercise without elicit a higher cardiovascular overload compared with split routine, however, caution should be taken when interpreting and applying these results to the general public, manly in cardiac patients, once our study was conducted in healthy trained men.

Despite the importance of our results, some limitations must be considered. First, we evaluated one mechanism of blood pressure control, there are other local mechanisms such as tissues releasing vasodilator substances and vasodilator response from contracting skeletal muscles during exercises which could be investigated. Second, we analyzed heart rate variability and blood pressure only during $30 \mathrm{~min}$ after exercise. In this way, we suggest future research to analyze the comparison between acute and chronic full versus split body routines on resting blood pressure and heart rate variability in patients with cardiovascular diseases and which measure other mechanisms of blood flow control.

In summary, PEH was influenced by the amount of muscle mass involved in a bout of $\mathrm{RE}$, as the $\mathrm{FB}$ condition induced lower 
SBP in relation to the UB after 30 min of recovery, as well as greater postexercise reduction in diastolic and MBP compared with the LB condition. In addition, there was a progressive increase in parasympathetic modulation during recovery and a sympathetic predominance that persisted until $30 \mathrm{~min}$ after exercise when compared to rest in all conditions. From a practical point of view, a full body routine could be used to potentiate blood pressure reduction after a RE program without elicit a higher cardiovascular overload compared with split body routine in health subjects.

\section{CONFLICT OF INTEREST}

No potential conflict of interest relevant to this article was reported.

\section{ACKNOWLEDGMENTS}

The authors would like to thank Erico Chagas Caperuto, Ph.D. from São Judas Tadeu University, São Paulo, Brazil for his support.

\section{REFERENCES}

Al Haddad H, Laursen PB, Chollet D, Ahmaidi S, Buchheit M. Reliability of resting and postexercise heart rate measures. Int J Sports Med 2011; 32:598-605.

American College of Sports Medicine. ACSM's guidelines for exercise testing and prescription. 7th ed. Philadelphia (PA): Lippincott Williams \& Wilkins; 2006.

Barbosa MP, da Silva NT, de Azevedo FM, Pastre CM, Vanderlei LC. Comparison of Polar ${ }^{\circledR}$ RS800G3 ${ }^{\mathrm{TM}}$ heart rate monitor with Polar $^{\circledR}$ $\mathrm{S} 810 \mathrm{i}^{\mathrm{TM}}$ and electrocardiogram to obtain the series of RR intervals and analysis of heart rate variability at rest. Clin Physiol Funct Imaging 2016:36:112-117.

Borresen J, Lambert MI. Autonomic control of heart rate during and after exercise: measurements and implications for monitoring training status. Sports Med 2008;38:633-646.

Coleman A, Freeman P, Steel S, Shennan A. Validation of the Omron MX3 Plus oscillometric blood pressure monitoring device according to the European Society of Hypertension international protocol. Blood Press Monit 2005;10:165-168.

de Salles BF, Maior AS, Polito M, Novaes J, Alexander J, Rhea M, Simão R. Influence of rest interval lengths on hypotensive response after strength training sessions performed by older men. J Strength Cond Res 2010;
24:3049-3054.

Di Blasio A, Sablone A, Civino P, D’Angelo E, Gallina S, Ripari P. Arm vs. Combined leg and arm exercise: blood pressure responses and ratings of perceived exertion at the same indirectly determined heart rate. J Sports Sci Med 2009;8:401-409.

Drouet PC, Archer DC, Munger CN, Coburn JW, Costa PB, Bottaro M, Brown LE. Hypotensive effects following upper vs. lower body resistance exercise between normotensive and prehypertensive men. J Exerc Physiol Online 2017;20:17-27.

Figueiredo T, Rhea MR, Peterson M, Miranda H, Bentes CM, dos Reis VM, Simão R. Influence of number of sets on blood pressure and heart rate variability after a strength training session. J Strength Cond Res 2015;29:1556-1563.

Gomes Anunciação P, Doederlein Polito M. A review on post-exercise hypotension in hypertensive individuals. Arq Bras Cardiol 2011;96: e100-109.

Halliwill JR. Mechanisms and clinical implications of post-exercise hypotension in humans. Exerc Sport Sci Rev 2001;29:65-70.

Heffernan KS, Kelly EE, Collier SR, Fernhall B. Cardiac autonomic modulation during recovery from acute endurance versus resistance exercise. Eur J Cardiovasc Prev Rehabil 2006;13:80-86.

Heffernan KS, Sosnoff JJ, Jae SY, Gates GJ, Fernhall B. Acute resistance exercise reduces heart rate complexity and increases QTc interval. Int J Sports Med 2008;29:289-293.

Hurley BF, Roth SM. Strength training in the elderly: effects on risk factors for age-related diseases. Sports Med 2000;30:249-268.

Joyner MJ, Casey DP. Regulation of increased blood flow (hyperemia) to muscles during exercise: a hierarchy of competing physiological needs. Physiol Rev 2015;95:549-601.

Kingsley JD, Hochgesang S, Brewer A, Buxton E, Martinson M, Heidner $\mathrm{G}$. Autonomic modulation in resistance-trained individuals after acute resistance exercise. Int J Sports Med 2014;35:851-856.

Kiviniemi AM, Hautala AJ, Kinnunen H, Nissilä J, Virtanen P, Karjalainen J, Tulppo MP. Daily exercise prescription on the basis of HR variability among men and women. Med Sci Sports Exerc 2010;42:13551363.

Kulics JM, Collins HL, DiCarlo SE. Postexercise hypotension is mediated by reductions in sympathetic nerve activity. Am J Physiol 1999;276(1 Pt 2):H27-32.

Lichtenstein AH, Appel LJ, Brands M, Carnethon M, Daniels S, Franch HA, Franklin B, Kris-Etherton P, Harris WS, Howard B, Karanja N, Lefevre M, Rudel L, Sacks F, Van Horn L, Winston M, Wylie-Rosett J. Diet and lifestyle recommendations revision 2006: a scientific statement from the American Heart Association Nutrition Committee. Circulation 2006;114:82-96. 
Mortensen SP, Saltin B. Regulation of the skeletal muscle blood flow in humans. Exp Physiol 2014;99:1552-1558.

Pescatello LS, Franklin BA, Fagard R, Farquhar WB, Kelley GA, Ray CA; American College of Sports Medicine. American College of Sports Medicine position stand. Exercise and hypertension. Med Sci Sports Exerc 2004;36:533-553.

Pokorný J, Staněk V, Vrána M. Sudden cardiac death thirty years ago and at present. The role of autonomic disturbances in acute myocardial infarction revisited. Physiol Res 2011;60:715-728.

Polito MD, Farinatti PT. The effects of muscle mass and number of sets during resistance exercise on postexercise hypotension. J Strength Cond Res 2009;23:2351-2357.

Rossi FE, Gerosa-Neto J, Zanchi NE, Cholewa JM, Lira FS. Impact of short and moderate rest intervals on the acute immunometabolic response to exhaustive strength exercise: Part I. J Strength Cond Res 2016;30: 1563-1569.

Simão R, Fleck SJ, Polito M, Monteiro W, Farinatti P. Effects of resistance training intensity, volume, and session format on the postexercise hypotensive response. J Strength Cond Res 2005;19:853-858.

Stanley J, Peake JM, Buchheit M. Cardiac parasympathetic reactivation following exercise: implications for training prescription. Sports Med 2013;43:1259-1277.

Vanderlei LC, Pastre CM, Hoshi RA, Carvalho TD, Godoy MF. Basic notions of heart rate variability and its clinical applicability. Rev Bras Cir Cardiovasc 2009;24:205-217.

Vanderlei LC, Silva RA, Pastre CM, Azevedo FM, Godoy MF. Comparison of the Polar S810i monitor and the ECG for the analysis of heart rate variability in the time and frequency domains. Braz J Med Biol Res 2008;41:854-859.

Vinik AI, Maser RE, Ziegler D. Autonomic imbalance: prophet of doom or scope for hope? Diabet Med 2011;28:643-651.

Volders PG. Novel insights into the role of the sympathetic nervous system in cardiac arrhythmogenesis. Heart Rhythm 2010;7:1900-1906. 